\title{
Estimating Beta $(\beta)$ Values of Stocks in the Creation of Diversified Portfolio - A Detailed Study
}

\author{
Syed Mohammad Faisal ${ }^{1}$, Ahmad Khalid Khan ${ }^{1}$, Omar Abdullah Al Aboud ${ }^{1}$ \\ ${ }^{1}$ Assistant professor, Kingdom of Saudi Arabia \\ Correspondence: Ahmad Khalid Khan, Department of Accounting, Faculty of Management, Jazan University, \\ Kingdom of Saudi Arabia.
}

Received: April 7, 2018

Accepted: April 25, 2018

Available online: April 26, 2018

doi:10.11114/aef.v5i3.3243

URL: https://doi.org/10.11114/aef.v5i3.3243

\begin{abstract}
In this paper researchers investigate thorough analysis of stocks from different sectors in order to estimate beta values and thus creating optimum portfolio of estimated low $\beta$ values.

There are many traditional as well modern stock market theories prevalent in the system to facilitate common investors to enhance their returns from the stock market. Investors usually only focus on expected returns from their investments in the stock market and the forego various types of systematic and unsystematic risks involved in their investments in stock market that is basically risky way for investment.

Therefore this paper is an attempt to inculcate some basic as well as advance knowledge to create awareness about various types of risks involved in their investments. In this paper we, researchers have considered beta to be measured of different stocks taken from various sectors in the stock market.
\end{abstract}

Keywords: beta, risk and return, investment, portfolio, CAPM

\section{Introduction}

Risk of any class whether controllable or un controllable can't be avoided in nature but can be reduced by diversification of portfolio specifically in share market investment.Each investors before making investment in share market needs know some basic information about Risk, types of risks, and other basic information about their investment. In that regard many investment theories were brought to their notice amongst them one theory known as Capital Asset Pricing Model (CAPM) initially developed by William Sharpe became very famous that best described the use of beta (systematic risk), Rf (risk free rate) and overall market rate of return in order to calculate required rate of return from the stock.

He gave following formula to calculate to know required rate

$\mathrm{Rr} \%=\mathrm{Rf}+[\beta \times(\mathrm{Mr}-\mathrm{Rf})]$

Where $\mathrm{Rr}>$ Required rate of return

Beta $(\beta)$ range from -1 to +1

Mr> Market rate of return

$\mathrm{Rf}>$ Risk free rate

But this model is workable if beta of security is known and if not then it is recommended to ascertain beta of stock to be familiar with whether to include that stock in portfolio or not to take account of in portfolio.

In this paper researchers will give their best expertise understanding about estimating of beta to make investors aware of their systematic risk with the following conjecture as mentioned below:

If $\beta>1$ then it is expected that volatility of stock will be more than market.

$\beta \quad<1$ then it is expected that volatility of stock will be less than market.

$\beta \quad=1$ it is expected that volatility of stock will move with the market.

Let's take one example to understand $\beta$ that if stock of particular stock is found to be 1.2 then stock is considered $20 \%$ 
more volatile than stock market as a whole.

In more simplified version that if market moves $10 \%$ then particular stock tends to move up by $12 \%$ i.e. more volatility of stock and this will be applied to all betas.

\section{Need for Study}

In today's parlance each and every investor tries to magnify their returns by investing in risky assets since it is based on one basic rule i. e. Higher the Risk, Higher the Return.

But in such investments merely commitment of money is not sufficient but it requires at least some basic knowledge about analysis of stocks. Investors need know some stock market theories based on various types of risks and returns involved in stocks.

That is why researchers were enthused and motivated to bring some common understanding to ascertain $\beta$ in various stocks amongst different sectors.

Here in this research paper we have been aiming at helping novice but high risk taking investors to construct such portfolio by estimating $\beta$ values to assess various risk factors usually uncontrollable by nature to enhance their returns from their investment.

Researchers by doing in depth analysis have selected therefore three booming sectors in the Indian stock market (BSE) such as- Healthcare, Software and Banking.

We further assume that aforesaid sectors are one of the best performing sectors and hence picked 3 performing companies from each sector depending from their historical performances in the stock market.

\section{Objective of Research}

- $\quad$ Applying CAPM to estimate $\beta$ values of various stocks

- $\quad$ To pick 9 stocks from 3 different sectors and analyze their $\beta$ values

- To build up diversified portfolio by picking just 5 companies with their lower $\beta$ s.

\section{Literature Review}

Ante Parkovic (2011) stated that CAPM was one of most trusted financial economy models highlighted various aspects of risk and its estimation. He further explained in his paper that stocks with higher beta gave higher returns than stocks with lower beta value. But further concluded in his research paper that beta alone can't be considered for creating diversified portfolio. Andrea Frazzini (2013) described same Capital Asset Pricing Model and later gave relationship between BAB ( Betting Against Beta) factor and low beta securities as well as high beta securities in the model that he proposed. In his research he gave concept of leveraged portfolios and unleveraged portfolios to suit risk preferences of investors. Omar (2017) gave another theory based on Sharpe Index Model and discussed excess to return beta ratio and concluded better concept than Markowitz theory of risk and return of portfolio. Nikolaos G. Theriou (2016) discussed in his research paper that all returns on stocks tend to move with systematic risks i.e. beta values of stocks. In his paper he conceptualized CAPM and studied Athens stock exchange and included financial and insurance companies listed in the concerned stock market and interpret results by estimating beta values of such companies in aforesaid sectors. Bali \& Robert (2016) in their paper elaborated the significance of dynamic conditional beta and daily returns of stocks. They also analyzed that stocks with high beta value have chances to yield high returns than low beta values that have low chances to yield returns from securities. Wiesław, Dębski and Świderski (2016) in their research paper explained various phases of stock market and analyzed movements of beta values in Bullish and Bearish market. In their paper they thoroughly studied 134 different stocks and their beta values during 2005-13 and thus gave in depth interpretation about bull and bear market with respect of their beta values movements. Another cited research paper in which Yin Foo(2016) stated about sharia and non sharia portfolio of securities and then compared their betas in bull as well bear market. Brennan (1988) gave absolutely brilliant theory about stock movements after split and then their changes in beta values, this proven insights in estimating beta of various stocks in simple manner for investors. Banz, R.W. (1981) argued that not only risk and return on stocks have relationship but also market value of stocks traded in market and their return in terms of their trading in secondary market as well as return in terms of dividend and bonuses. Singh, R. (2008) discussed in his research that beta is fundamental unit to measure financial and investment portfolios, later he also gave conclusion that fewer studies done so far to ascertain beta coefficient to analyze investment portfolio.

While going through number of articles and research papers, contributions of so many scholars like Blume, M.E. (1971), Bhaduri, S. Durai, S. (2006), Benson, P.G. (1982), Deb, Misra, S. (2011) in their work in estimating beta values can't be denied and recommended to study for researchers . 


\section{Industry and Company Analysis for Beta Calculations}

Researchers have done their own criterion for selection of sectors and respective companies to construct optimum diversified portfolio with respect to their lowered betas.

Following conditions were carefully monitored for the selection of Industries and Companies as mentioned below-

- Fundamental Analysis of economy for selection of scrips from different sectors,

- Market Capitalization, EPS and P/E ratios of concerned companies(S/W, Healthcare and Infrastructure ) three companies taken from each sector

- 10 years index return(SENSEX) and companies' returns

\section{Research Methodology}

\begin{tabular}{l|l}
\hline Particulars & Description \\
\hline Sample Size & $\begin{array}{l}\text { Sectors :- 3 sectors taken from the Index i.e. Software, Healthcare, and Infrastructure } \\
\text { Companies :- 9 companies taken } \\
\text { Software ( Wipro, TCS, Infosys), } \\
\text { Healthcare( Sun, Cipla, Glaxo), } \\
\text { Infrastructure(Siemen, L\& T,ABB) }\end{array}$ \\
\hline Data Collection & $\begin{array}{l}\text { Industries and respective companies are selected on industry, company and other required analyses } \\
\text { in which 10 years data taken for our study }\end{array}$ \\
\hline Research Design & Descriptive and Quantitative \\
\hline Sources of Data & $\begin{array}{l}\text { Secondary data collected through money control and BSE India (10 Years data from 2009 -2018 } \\
\text { (Feb) taken for study) }\end{array}$ \\
\hline $\begin{array}{l}\text { Method } \\
\text { calculation of } \beta\end{array}$ & for \\
\hline Data Analysis & Covariance of two assets i.e. Stock and Market Index \\
\hline Covariance, Spearman's Correlation, Regression
\end{tabular}

\section{Significance of Data of Companies in Software Sector}

\begin{tabular}{|r|r|r|r|r|r|r|r|}
\hline Year & Infosys & SENSEX & $\boldsymbol{X}_{R a}$ & $\boldsymbol{X}_{R a}-\boldsymbol{M}_{\boldsymbol{x}}$ & $\boldsymbol{Y}_{R a}$ & $\boldsymbol{Y}_{R a}-\boldsymbol{M}_{\boldsymbol{y}}$ & $\begin{array}{r}\text { Sum } \\
\text { Diffs }\end{array}$ \\
\hline 2009 & 651.32 & 17464.81 & 2 & -3.5 & 2 & -3.5 & 12.25 \\
\hline 2010 & 861.25 & 20509.09 & 4 & -1.5 & 4 & -1.5 & 2.25 \\
\hline 2011 & 691.27 & 15454.92 & 3 & -2.5 & 1 & -4.5 & 11.25 \\
\hline 2012 & 579.63 & 19426.71 & 1 & -4.5 & 1 & -2.5 & 11.25 \\
\hline 2013 & 871.38 & 21170.68 & 5 & -0.5 & 3 & -0.5 & 0.25 \\
\hline 2014 & 985.6 & 27499.42 & 6 & 0.5 & 8 & 2.5 & 1.25 \\
\hline 2015 & 1104.55 & 26117.54 & 9 & 3.5 & 6 & 0.5 & 1.75 \\
\hline 2016 & 1010.7 & 26626.46 & 7 & 1.5 & 7 & 1.5 & 2.25 \\
\hline 2017 & 1039.3 & 34056.83 & 8 & 2.5 & 9 & 3.5 & 8.75 \\
\hline 2018 & 1108.45 & 34195.94 & 10 & 4.5 & 10 & 4.5 & 20.25 \\
\hline
\end{tabular}

\begin{tabular}{|l|}
\hline Results Details \\
\hline X Ranks \\
\hline Mean: 5.5 \\
\hline Standard Dev: 3.03 \\
\hline Y Ranks \\
\hline Mean: 5.5 \\
\hline Standard Dev: 3.03 \\
\hline Combined Covariance $=71.5 / 9=7.94$ \\
\hline $\mathrm{R}=7.94 /(3.03 * 3.03)=0.867$ \\
\hline
\end{tabular}

The value of $\mathrm{R}$ is 0.867 . By normal standards, the association between the two variables would be considered statistically significant. 


\begin{tabular}{|r|r|r|r|r|r|r|r|}
\hline Year & Wipro & SENSEX & $X_{R a}$ & $X_{R a}-M_{x}$ & $Y_{R a}$ & $Y_{R a}-M_{y}$ & Sum Diffs \\
\hline 2009 & 203.8 & 17464.81 & 3 & -2.5 & 2 & -3.5 & 8.75 \\
\hline 2010 & 245.1 & 20509.09 & 5 & -0.5 & 4 & -1.5 & 0.75 \\
\hline 2011 & 199.4 & 15454.92 & 2 & -3.5 & 1 & -4.5 & 15.75 \\
\hline 2012 & 197.2 & 19426.71 & 1 & -4.5 & 3 & -2.5 & 11.25 \\
\hline 2013 & 279.5 & 21170.68 & 7 & 1.5 & 5 & -0.5 & -0.75 \\
\hline 2014 & 276.9 & 27499.42 & 6 & 0.5 & 8 & 2.5 & 1.25 \\
\hline 2015 & 279.9 & 26117.54 & 8 & 2.5 & 6 & 0.5 & 1.25 \\
\hline 2016 & 237 & 26626.46 & 4 & -1.5 & 7 & 1.5 & -2.25 \\
\hline 2017 & 313.4 & 34056.83 & 10 & 4.5 & 9 & 3.5 & 15.75 \\
\hline 2018 & 294.9 & 34195.94 & 9 & 3.5 & 10 & 4.5 & 15.75 \\
\hline
\end{tabular}

\begin{tabular}{|l|}
\hline Results Details \\
\hline X Ranks \\
\hline Mean: 5.5 \\
\hline Standard Dev: 3.03 \\
\hline Y Ranks \\
\hline Mean: 5.5 \\
\hline Standard Dev: 3.03 \\
\hline Combined Covariance $=67.5 / 9=7.5$ \\
\hline $\mathrm{R}=7.5 /(3.03 * 3.03)=0.818$ \\
\hline
\end{tabular}

The value of $\mathrm{R}$ is 0.818 . By normal standards, the association between the two variables would be considered statistically significant.

\begin{tabular}{|l|r|r|r|r|r|r|r|}
\hline Year & TCS & SENSEX & $X_{R a}$ & $X_{R a}-M_{x}$ & $Y_{R a}$ & $Y_{R a}-M_{y}$ & Sum Diffs \\
\hline 2009 & 749.75 & 17464.81 & 1 & -4.5 & 2 & -3.5 & 15.75 \\
\hline 2010 & 1165.1 & 20509.09 & 3 & -2.5 & 4 & -1.5 & 3.75 \\
\hline 2011 & 1161.3 & 15454.92 & 2 & -3.5 & 1 & -4.5 & 15.75 \\
\hline 2012 & 1258.6 & 19426.71 & 4 & -1.5 & 3 & -2.5 & 3.75 \\
\hline 2013 & 2171 & 21170.68 & 5 & -0.5 & 5 & -0.5 & 0.25 \\
\hline 2014 & 2554.7 & 27499.42 & 8 & 2.5 & 8 & 2.5 & 6.25 \\
\hline 2015 & 2439.2 & 26117.54 & 7 & 1.5 & 5 & 0.5 & 0.75 \\
\hline 2016 & 2362 & 26626.46 & 6 & 0.5 & 7 & 1.5 & 0.75 \\
\hline 2017 & 2700.4 & 34056.83 & 9 & 3.5 & 9 & 3.5 & 12.25 \\
\hline 2018 & 2995.4 & 34195.94 & 10 & 4.5 & 10 & 4.5 & 20.25 \\
\hline
\end{tabular}

\begin{tabular}{|l|}
\hline Result Details \\
\hline X Ranks \\
\hline Mean: 5.5 \\
\hline Standard Dev: 3.03 \\
\hline Y Ranks \\
\hline Mean: 5.5 \\
\hline Standard Dev: 3.03 \\
\hline Combined Covariance $=79.5 / 9=8.83$ \\
\hline$R=8.83 /\left(3.03^{*} 3.03\right)=0.964$ \\
\hline
\end{tabular}

The value of $\mathrm{R}$ is 0.964 . By normal standards, the association between the two variables would be considered statistically significant 


\begin{tabular}{|r|r|r|r|r|r|r|r|}
\hline \multicolumn{1}{l|}{ Year } & Sun Pharmaceuticals & \multicolumn{1}{l|}{ SENSEX } & $X_{R a}$ & $X_{R a}-M_{x}$ & $Y_{R a}$ & $Y_{R a}-M y$ & Sum Diffs \\
\hline 2009 & 150.71 & 17464.81 & 1 & -4.5 & 2 & -3.5 & 15.75 \\
\hline 2010 & 242.33 & 20509.09 & 2 & -3.5 & 4 & -1.5 & 5.25 \\
\hline 2011 & 248.43 & 15454.92 & 3 & -2.5 & 1 & -4.5 & 11.25 \\
\hline 2012 & 367.75 & 19426.71 & 4 & -1.5 & 3 & -2.5 & 3.75 \\
\hline 2013 & 567.75 & 21170.68 & 6 & 0.5 & 5 & -0.5 & -0.25 \\
\hline 2014 & 826.15 & 27499.42 & 10 & 4.5 & 8 & 2.5 & 11.25 \\
\hline 2015 & 819.95 & 26117.54 & 9 & 3.5 & 6 & 0.5 & 1.75 \\
\hline 2016 & 629.75 & 26626.46 & 6 & 2.5 & 7 & 1.5 & 3.75 \\
\hline 2017 & 570.8 & 34056.83 & 7 & 1.5 & 9 & 3.5 & 5.25 \\
\hline 2018 & 551.9 & 34195.94 & 5 & -0.5 & 10 & 4.5 & -2.25 \\
\hline
\end{tabular}

\begin{tabular}{|l|}
\hline$\underline{\text { Result Details }}$ \\
\hline X Ranks \\
\hline Mean: 5.5 \\
\hline Standard Dev: 3.03 \\
\hline Y Ranks \\
\hline Mean: 5.5 \\
\hline Standard Dev: 3.03 \\
\hline Combined Covariance $=55.5 / 9=6.17$ \\
\hline $\mathrm{R}=6.17 /(3.03 * 3.03)=0.673$ \\
\hline
\end{tabular}

The value of $\mathrm{R}$ is 0.673 . By normal standards, the association between the two variables would be considered statistically significant.

\begin{tabular}{|l|r|r|r|r|r|r|r|}
\hline Year & Cipla & SENSEX & $X_{R a}$ & $X_{R a}-M_{x}$ & $Y_{R a}$ & $Y_{R a}-M_{y}$ & Sum Diffs \\
\hline 2009 & 335.6 & 17464.81 & 2 & -3.5 & 2 & -3.5 & 12.25 \\
\hline 2010 & 369.9 & 20509.09 & 3 & -2.5 & 4 & -1.5 & 3.75 \\
\hline 2011 & 319.6 & 15454.92 & 1 & -4.5 & 1 & -4.5 & 20.25 \\
\hline 2012 & 414.1 & 19426.71 & 5 & -0.5 & 3 & -2.5 & 1.25 \\
\hline 2013 & 400.6 & 21170.68 & 4 & -1.5 & 5 & -0.5 & 0.75 \\
\hline 2014 & 625.8 & 27499.42 & 9 & 3.5 & 8 & 2.5 & 8.75 \\
\hline 2015 & 649.5 & 26117.54 & 10 & 4.5 & 6 & 0.5 & 2.25 \\
\hline 2016 & 568.2 & 26626.46 & 7 & 1.5 & 7 & 1.5 & 2.25 \\
\hline 2017 & 607.2 & 34056.83 & 8 & 2.5 & 9 & 3.5 & 8.75 \\
\hline 2018 & 565.7 & 34195.94 & 6 & 0.5 & 10 & 4.5 & 2.25 \\
\hline
\end{tabular}

\begin{tabular}{|l|}
\hline Result Details \\
\hline$X$ Ranks \\
\hline Mean: 5.5 \\
\hline Standard Dev: 3.03 \\
\hline YRanks \\
\hline Mean: 5.5 \\
\hline Standard Dev: 3.03 \\
\hline Combined Covariance $=62.5 / 9=6.94$ \\
\hline $\mathrm{R}=6.94 /(3.03 * 3.03)=0.758$ \\
\hline
\end{tabular}

The value of $\mathrm{R}$ is 0.758 . By normal standards, the association between the two variables would be considered statistically significant. 


\begin{tabular}{|c|c|c|c|c|c|c|c|}
\hline Year & GlaxoSmithKline & SENSEX & $X R a$ & $X R a-M x$ & $Y R a$ & $Y R a-M y$ & Sum Diffs \\
\hline 2009 & 1610 & 17464.8 & 1 & -4.5 & 2 & -3.5 & 15.75 \\
\hline 2010 & 2342.6 & 20509.1 & 4 & -1.5 & 4 & -1.5 & 2.25 \\
\hline 2011 & 1936.5 & 15454.9 & 2 & -3.5 & 1 & -4.5 & 15.75 \\
\hline 2012 & 2165.7 & 19426.7 & 3 & -2.5 & 3 & -2.5 & 6.25 \\
\hline 2013 & 2992.6 & 21170.7 & 8 & 2.5 & 5 & -0.5 & -1.25 \\
\hline 2014 & 3196.75 & 27499.4 & 9 & 3.5 & 8 & 2.5 & 8.75 \\
\hline 2015 & 3321.05 & 26117.5 & 10 & 4.5 & 6 & 0.5 & 2.25 \\
\hline 2016 & 2730.35 & 26626.5 & 7 & 1.5 & 7 & 1.5 & 2.25 \\
\hline 2017 & 2480.9 & 34056.8 & 6 & 0.5 & 9 & 3.5 & 1.75 \\
\hline 2018 & 2450.65 & 34195.9 & 5 & -0.5 & 10 & 4.5 & -2.25 \\
\hline
\end{tabular}

\begin{tabular}{|l|}
\hline Result Details \\
\hline X Ranks \\
\hline Mean: 5.5 \\
\hline Standard Dev: 3.03 \\
\hline Y Ranks \\
\hline Mean: 5.5 \\
\hline Standard Dev: 3.03 \\
\hline Combined Covariance $=51.5 / 9=5.72$ \\
\hline $\mathrm{R}=5.72 /(3.03 * 3.03)=0.624$ \\
\hline
\end{tabular}

The value of $\mathrm{R}$ is 0.624 . By normal standards, the association between the two variables would be considered statistically significant.

\begin{tabular}{|r|r|r|r|r|r|r|r|}
\hline \multicolumn{1}{l|}{ Year } & Siemens & SENSEX & $X R a$ & \multicolumn{1}{|c|}{ XRa - Mx } & YRa & YRa - My & Sum Diffs \\
\hline 2009 & 582.15 & 17464.81 & 1 & -4.5 & 2 & -3.5 & 15.75 \\
\hline 2010 & 820.75 & 20509.09 & 5 & -0.5 & 4 & -1.5 & 0.75 \\
\hline 2011 & 642.3 & 15454.92 & 2 & -3.5 & 1 & -4.5 & 15.75 \\
\hline 2012 & 667 & 19426.71 & 4 & -1.5 & 3 & -2.5 & 3.75 \\
\hline 2013 & 663.7 & 21170.68 & 3 & -2.5 & 5 & -0.5 & 1.25 \\
\hline 2014 & 907.05 & 27499.42 & 6 & 0.5 & 8 & 2.5 & 1.25 \\
\hline 2015 & 1200.8 & 26117.54 & 8 & 2.5 & 6 & 0.5 & 1.25 \\
\hline 2016 & 1112.8 & 26626.46 & 7 & 1.5 & 7 & 1.5 & 2.25 \\
\hline 2017 & 1236.8 & 34056.83 & 9 & 3.5 & 9 & 3.5 & 12.25 \\
\hline 2018 & 1251.5 & 34195.94 & 10 & 4.5 & 10 & 4.5 & 20.25 \\
\hline
\end{tabular}

\begin{tabular}{|l|}
\hline Result Details \\
\hline$X$ Ranks \\
\hline Mean: 5.5 \\
\hline Standard Dev: 3.03 \\
\hline Y Ranks \\
\hline Mean: 5.5 \\
\hline Standard Dev: 3.03 \\
\hline Combined Covariance $=74.5 / 9=8.28$ \\
\hline $\mathrm{R}=8.28 /(3.03 * 3.03)=0.903$ \\
\hline
\end{tabular}

The value of $\mathrm{R}$ is 0.90303 . By normal standards, the association between the two variables would be considered statistically significant. 


\begin{tabular}{|r|r|r|r|r|r|r|r|}
\hline Year & L\&T & SENSEX & $X_{R a}$ & $X_{R a}-M_{x}$ & $Y_{R a}$ & $Y_{R a}-M_{y}$ & Sum Diffs \\
\hline 2009 & 750.17 & 17464.81 & 4 & -1.5 & 2 & -3.5 & 5.25 \\
\hline 2010 & 884.02 & 20509.09 & 6 & 0.5 & 4 & -1.5 & -0.75 \\
\hline 2011 & 444.50 & 15454.92 & 1 & -4.5 & 1 & -4.5 & 20.25 \\
\hline 2012 & 707.32 & 19426.71 & 2 & -3.5 & 3 & -2.5 & 8.75 \\
\hline 2013 & 713.30 & 21170.68 & 3 & -2.5 & 5 & -0.5 & 1.25 \\
\hline 2014 & 997.72 & 27499.42 & 8 & 2.5 & 8 & 2.5 & 6.25 \\
\hline 2015 & 850.48 & 26117.54 & 5 & -0.5 & 6 & 0.5 & -0.25 \\
\hline 2016 & 899.64 & 26626.46 & 7 & 1.5 & 7 & 1.5 & 2.25 \\
\hline 2017 & $1,256.95$ & 34056.83 & 9 & 3.5 & 9 & 3.5 & 12.25 \\
\hline 2018 & $1,354.00$ & 34195.94 & 10 & 4.5 & 10 & 4.5 & 20.25 \\
\hline
\end{tabular}

\begin{tabular}{|l|}
\hline Result Details \\
\hline$X$ Ranks \\
\hline Mean: 5.5 \\
\hline Standard Dev: 3.03 \\
\hline Y Ranks \\
\hline Mean: 5.5 \\
\hline Standard Dev: 3.03 \\
\hline Combined Covariance $=75.5 / 9=8.39$ \\
\hline $\mathrm{R}=8.39 /\left(3.03^{*} 3.03\right)=0.915$ \\
\hline
\end{tabular}

The value of $\mathrm{R}$ is 0.915 . By normal standards, the association between the two variables would be considered statistically significant.

\begin{tabular}{|l|r|r|r|r|r|r|r|}
\hline Year & ABB & SENSEX & $X_{R a}$ & $X_{R a}-M_{x}$ & $Y_{R a}$ & $Y_{R a}-M_{y}$ & Sum Diffs \\
\hline 2009 & 767.25 & 17464.81 & 4 & -1.5 & 2 & -3.5 & 5.25 \\
\hline 2010 & 792.25 & 20509.09 & 5 & -0.5 & 4 & -1.5 & 0.75 \\
\hline 2011 & 583.60 & 15454.92 & 1 & -4.5 & 1 & -4.5 & 20.25 \\
\hline 2012 & 700.40 & 19426.71 & 3 & -2.5 & 3 & -2.5 & 6.25 \\
\hline 2013 & 692.80 & 21170.68 & 2 & -3.5 & 5 & -0.5 & 1.75 \\
\hline 2014 & $1,286.20$ & 27499.42 & 8 & 2.5 & 8 & 2.5 & 6.25 \\
\hline 2015 & $1,118.10$ & 26117.54 & 7 & 1.5 & 6 & 0.5 & 0.75 \\
\hline 2016 & $1,039.10$ & 26626.46 & 6 & 0.5 & 7 & 1.5 & 0.75 \\
\hline 2017 & $1,399.50$ & 34056.83 & 9 & 3.5 & 9 & 3.5 & 12.25 \\
\hline 2018 & $1,581.45$ & 34195.94 & 10 & 4.5 & 10 & 4.5 & 20.25 \\
\hline
\end{tabular}

\begin{tabular}{|l|}
\hline Result Details \\
\hline$X$ Ranks \\
\hline Mean: 5.5 \\
\hline Standard Dev: 3.03 \\
\hline$Y$ Ranks \\
\hline Mean: 5.5 \\
\hline Standard Dev: 3.03 \\
\hline Combined Covariance $=74.5 / 9=8.28$ \\
\hline $\mathrm{R}=8.28 /(3.03 * 3.03)=0.903$ \\
\hline
\end{tabular}

The value of $\mathrm{R}$ is 0.903 . By normal standards, the association between the two variables would be considered statistically significant. 
Let's now take a look frequency table of Sensex data range for the period of 10 years

\begin{tabular}{|l|r|}
\hline \multicolumn{2}{|c|}{ Frequency Table of Sensex } \\
\hline \multicolumn{1}{|c|}{ Sensex } & \multicolumn{1}{c|}{ Count } \\
\hline $15000-20499.99$ & 2 \\
\hline $20500-25999.99$ & 3 \\
\hline $26000-31499.99$ & 2 \\
\hline $31500-36999.99$ & 24252.24 \\
\hline \multicolumn{2}{|c|}{ Your Histogram } \\
\hline Mean & 6539.81453 \\
\hline Standard Deviation (s) & 15454.92 \\
\hline Lowest Score & 34195.94 \\
\hline Highest Score & 18741.02 \\
\hline Distribution Range & 10 \\
\hline Total Number of Scores & 10 \\
\hline Number of Distinct Scores & 15000 \\
\hline Lowest Class Value & 36999.99 \\
\hline Highest Class Value & 4 \\
\hline Number of Classes & 5500 \\
\hline Class Range & \\
\hline
\end{tabular}

\section{Analysis and Interpretations}

On the basis of three sectors and three companies from each sector taken in our study as mentioned in our research methodology we have used co variance between two assets i.e. stock and index in our study, betas have been calculated.

Following tables in each sector described respective beta. In the table 1 we have calculated $\beta$ for software sector and out of that three companies on the basis of market capitalization and $\mathrm{P} / \mathrm{E}$ Ratio have been taken into account. As we see that $\beta$ of Infosys, Wipro and TCS were found to be less than one $(\beta<1)$ that means selected stocks are less volatile than the current market and this will make investment feasible in those scrips in S/W sector and also it was found that $\beta$ value of Wipro is least volatile followed by Infosys and TCS and could be considered preferred stock in the created portfolio.

Table 1. $\beta$ Values of Companies in Software Sector

\begin{tabular}{|c|c|c|c|c|c|c|c|}
\hline Year & Infosys & Wipro & TCS & SENSEX & Infosys $\beta$ & Wipro $\beta$ & TCS Beta \\
\hline 2009 & 651.32 & 203.82 & 749.75 & 17464.81 & \multirow{10}{*}{0.022596262} & \multirow{10}{*}{0.00495928} & \multirow{10}{*}{0.098869275} \\
\hline 2010 & 861.25 & 245.13 & 1165.05 & 20509.09 & & & \\
\hline 2011 & 691.27 & 199.4 & 1161.25 & 15454.92 & & & \\
\hline 2012 & 579.63 & 197.18 & 1258.55 & 19426.71 & & & \\
\hline 2013 & 871.38 & 279.53 & 2170.95 & 21170.68 & & & \\
\hline 2014 & 985.6 & 276.9 & 2554.7 & 27499.42 & & & \\
\hline 2015 & 1104.55 & 279.9 & 2439.2 & 26117.54 & & & \\
\hline 2016 & 1010.7 & 237 & 2361.95 & 26626.46 & & & \\
\hline 2017 & 1039.3 & 313.4 & 2700.4 & 34056.83 & & & \\
\hline 2018 & 1108.45 & 294.9 & 2995.35 & 34195.94 & & & \\
\hline
\end{tabular}

Similarly we have calculated $\beta$ values for healthcare sector and thus three companies named Sun Pharmaceuticals, Cipla and Glaxo Smithcline were selected and their $\beta$ values calculated and found less volatile than overall market.

Also found that Cipla has emerged least volatile company with $\beta$ value of 0.015113532 from investment preference. 
Table 2. $\beta$ Values of Companies in Healthcare Sector

\begin{tabular}{|c|c|c|c|c|c|c|c|}
\hline Year & $\begin{array}{l}\text { Sun } \\
\text { Pharmaceuticals }\end{array}$ & Cipla & $\begin{array}{l}\text { Glaxo } \\
\text { SmithKline }\end{array}$ & SENSEX & $\begin{array}{l}\text { Sun } \\
\text { Pharmaceuticals } \\
\text { Beta }\end{array}$ & $\begin{array}{l}\text { Cipla } \\
\text { Beta }\end{array}$ & $\begin{array}{l}\text { Glaxo } \\
\text { SmithKline } \\
\text { Beta }\end{array}$ \\
\hline 2009 & 150.71 & 335.6 & 1610 & 17464.81 & \multirow{10}{*}{0.021398566} & \multirow{10}{*}{0.015113532} & \multirow{10}{*}{0.035879491} \\
\hline 2010 & 242.33 & 369.9 & 2342.6 & 20509.09 & & & \\
\hline 2011 & 248.43 & 319.55 & 1936.5 & 15454.92 & & & \\
\hline 2012 & 367.75 & 414.1 & 2165.7 & 19426.71 & & & \\
\hline 2013 & 567.75 & 400.55 & 2992.6 & 21170.68 & & & \\
\hline 2014 & 826.15 & 625.8 & 3196.75 & 27499.42 & & & \\
\hline 2015 & 819.95 & 649.5 & 3321.05 & 26117.54 & & & \\
\hline 2016 & 629.75 & 568.2 & 2730.35 & 26626.46 & & & \\
\hline 2017 & 570.8 & 607.15 & 2480.9 & 34056.83 & & & \\
\hline 2018 & 551.9 & 565.65 & 2450.65 & 34195.94 & & & \\
\hline
\end{tabular}

Similarly we have calculated $\beta$ values for infrastructure sector and thus three companies named Siemens, L\& T and ABB were selected and their $\beta$ values $(<1)$ calculated and found less volatile than overall market.

Also found that Siemens has emerged least volatile company with $\beta$ value of 0.033809924 from investment preference.

Table 3. $\beta$ Values of Companies in Infrastructure Sector

\begin{tabular}{|c|c|c|c|c|c|c|c|}
\hline Year & Siemens & L\& $T$ & $\begin{array}{l}\text { ABB } \\
\text { India }\end{array}$ & SENSEX & $\begin{array}{l}\text { Siemens } \\
\text { Beta }\end{array}$ & L\& T Beta & $\begin{array}{l}\text { ABB India } \\
\text { Beta }\end{array}$ \\
\hline 2009 & 582.15 & 750.17 & 767.25 & 17464.81 & \multirow{10}{*}{0.033809924} & \multirow{10}{*}{0.034668296} & \multirow{10}{*}{0.045252608} \\
\hline 2010 & 820.75 & 884.02 & 792.25 & 20509.09 & & & \\
\hline 2011 & 642.3 & 444.5 & 583.6 & 15454.92 & & & \\
\hline 2012 & 667 & 707.32 & 700.4 & 19426.71 & & & \\
\hline 2013 & 663.7 & 713.3 & 692.8 & 21170.68 & & & \\
\hline 2014 & 907.05 & 997.72 & 1286.2 & 27499.42 & & & \\
\hline 2015 & 1200.8 & 850.48 & 1118.1 & 26117.54 & & & \\
\hline 2016 & 1112.8 & 899.64 & 1039.1 & 26626.46 & & & \\
\hline 2017 & 1236.8 & 1256.95 & 1399.5 & 34056.83 & & & \\
\hline 2018 & 1251.5 & 1354 & 1581.45 & 34195.94 & & & \\
\hline
\end{tabular}

Now at the end of our analysis we researchers have ranked these below mentioned companies with respect to their lowest beta values with the purpose of investment while bearing lower risk and thus suggest including in the portfolio and allocating investment funds accordingly.

\begin{tabular}{|l|r|}
\hline Name of Companies & Ranking of Beta Values \\
\hline Wipro & 0.005 \\
\hline Cipla & 0.015 \\
\hline Sun Pharmaceuticals & 0.021 \\
\hline Infosys & 0.023 \\
\hline Siemens & 0.034 \\
\hline L\& T & 0.035 \\
\hline GlaxoSmithKline & 0.036 \\
\hline ABB India & 0.045 \\
\hline TCS & 0.099 \\
\hline$* * * 2018$ data as on Feb/18
\end{tabular}

\section{Limitations of Research}

During our research based study we have found certain unavoidable limitations as mentioned below- 
- Due to time constraint only 3 sectors selected

- $\quad$ Portfolio construction is based on only $\beta$ values as per Capital Asset Pricing Model

- $\quad$ Expected future uncertainties of stocks not considered Only historical data considered during stocks analysis

\section{Conclusion}

During our study we have made an attempt to focus the relevance of systematic risk factors and its measurement through calculations of $\beta$ values of stocks despite the fact that it is merely simple but crucial part of study.

To make investors (slightly risk takers or risk averse ) aware of role and significance of estimated $\beta$ values of stocks before doing investment.

We have drawn conclusion on the basis of Beta calculations of stocks for the purpose of monitoring performance for investors who do not have enough understanding of analysis but willing to invest in share market.

Researchers have carefully monitored and analyzed three sectors infrastructure, healthcare and software depending upon their huge demand in Indian market.

During our research we were quite aware about basic fundamental analysis of stocks such as industry as well company analysis as we did in our study.

\section{Scope of Further Study}

Although researchers have left no stone unturned to make this paper as panacea for novice investors who want moderate returns in stocks but due to their limited knowledge they were not able to dare to invest in stock market.

While writing this research paper many more advanced analysis were due but to see the length of the paper, researchers have not extended and elaborated their research and left plenty of scope for further study where to be more accurate in estimating risks (controllable as well uncontrollable) and diversification of companies with inclusion of Beta to Access Ratio along with some share market valuation models, dividend distribution theories such as Walter and Gordon's model and their role in investment in stocks to get higher returns with minimized returns in the further research.

\section{References}

Alexander, G. J., \& Benson, P. G. (1982). More on Beta as a Random Coefficient. Journal of Financial and Quantitative Analysis, 17(1), 27-36. https://doi.org/10.2307/2330927

Ante, P. (2011). Research of beta as adequate risk measure-is beta still alive? Faculty of Economics Split Croatian Operational Research Review (CRORR), 2, 2011.

Banz, R. W. (1981). The relationship between return and market value of common stocks, Journal of Financial Economics, 9(1), 3-18. https://doi.org/10.1016/0304-405X(81)90018-0

Bhaduri, S., \& Durai, S. (2006). Asymmetric beta in bull and bear market conditions: evidences from India, Applied Financial Economics Letters, 2, 55-59. https://doi.org/10.1080/17446540500396834

Blume, M. E. (1971). On the Assessment of Risk, Journal of Finance, 26(1), 1-10. https://doi.org/10.1111/j.1540-6261.1971.tb00584.x

Brennan, M. J., \& Copeland, T. E. (1988). Beta Changes around Stock Splits: A Note, Journal of Finance, 43(4), 10091013. https://doi.org/10.1111/j.1540-6261.1988.tb02618.x

Clarkson, P. M., \& Thompson, R. (1990). Empirical Estimates of Beta When Investors Face Estimation Risk, Journal of Finance, 45(2), 431-45. https://doi.org/10.1111/j.1540-6261.1990.tb03697.x

Deb, S. G., \& Misra, S. (2011). Are Equity Betas Stable? Evidence from Indian Equity Market. The IUP Journal of Applied Finance, 17(4), 5-25.

Faisal, K. A. A. (2018). Study of Managerial Decision Making Linked to Operating and Financial Leverage, International Journal of Accounting and Finance Research, 7(1), 139-143. https://doi.org/10.5430/afr.v7n1p139

Fama, E. F., \& French, K. R. (2004). The Capital Asset Pricing Model: Theory and Evidence. Journal of Economic Perspective, 18(3), 25-46. https://doi.org/10.1257/0895330042162430

Gordon, M. J. (1963). Optimal Investment and Financing Policy. Journal of Finance, 264-272.

James, W. (1963). Dividend Policy its effluence on the value of enterprise. Journals of Finance-18th May, 280.

Miller, M. H., \& Modigliani, F. (1961). Dividend Policy, Growth and the Valuation of Shares. Journal of Business, 34, 411-433. https://doi.org/10.1086/294442 
Nawrocki, D. (1999). A brief history of downside risk measures, Journal of Investing, 9-25. https://doi.org/10.3905/joi.1999.319365

Niarchos, N. A., \& Alexakis, C. A. (2000). The predictive power of macroeconomic variables on stock market returns: the case of the Athens stock exchange, Spoudai, 50, 74-86.

Nikolaos, G., Theriou, V. P. A., \& Dimitrios, I. M. (2007). Testing the relation between beta and returns in the Athens stock exchange.

Omar, A., \& Faisal. (2017). An Investigation Of Indian Security Market The Viewpoint Of FMCG Companies Who Voyage From Good To Great, International Journal of Economics, Commerce and Management, V(12). http://ijecm.co.uk/wpcontent/uploads/2017/12/51258.pdf

Perold, A. F. (2004). The Capital Asset Pricing Model. Journal of Economic Perspective, 18(3), 3-24. https://doi.org/10.1257/0895330042162340

Prasanna, C. (2010). Dividend policy and firm value, Financial Management, Theory and practice, Fifth edition, Tata McGraw-Hill Publishing Co., New Delhi.

Ross, S. (1976). The arbitrage theory of capital asset pricing, Journal of Economic Theory, 13(6), 341-60. https://doi.org/10.1016/0022-0531(76)90046-6

Shanken, J. (1985). Multivariate tests of the zero-beta CAPM, Journal of Financial Economics, 14, 327-348. https://doi.org/10.1016/0304-405X(85)90002-9

Sharpe, W. F. (1964). Capital asset prices: a theory of market equilibrium under conditions of risk, Journal of Finance, 19, 425-442.

Singh, R. (2008). Beta Stationarity over Bull and Bear Markets In India, The Icfai Journal of Applied Finance, 14(4), 3247.

Syed, O., \& Khalid. (2018). An Empirical Study of Technological Innovations in the Field of Accounting - Boon or Bane. Business and Management Studies, 4(1). https://doi.org/10.11114/bms.v4i1.3057

Wiesław, D., Ewa, F. S., \& Bartosz, Ś. (2016). Beta Stability Over Bull and Bear Market on the Warsaw Stock Exchange. The Journal of University of Szczecin. https://doi.org/10.1515/foli-2016-0006

Yin, F. C., \& Michelle, T. Z. L. (2016). Diversification: A Sharia Effect? Procedia - Social and Behavioral Sciences, 219, 193-200. https://doi.org/10.1016/j.sbspro.2016.05.005

\section{Copyrights}

Copyright for this article is retained by the author(s), with first publication rights granted to the journal.

This is an open-access article distributed under the terms and conditions of the Creative Commons Attribution license which permits unrestricted use, distribution, and reproduction in any medium, provided the original work is properly cited. 